\title{
Zwischen internationaler Verantwortung und neuer Friedenspolitik
}

\author{
Die ersten vier Jahre rot-grüner Außen- und Sicherheitspolitik: Eine Bilanz
}

\author{
Gernot Erler, MdB, Stellver- \\ tretender Vorsitzender der SPD- \\ Bundestagsfraktion und zustän- \\ dig für Außen-, Sicherheits-, \\ Entwicklungspolitik und Men- \\ schenrechte, Berlin
}

\section{Richtung halten in unruhi- ger Zeit: Eine Kurzüber- sicht}

Vier bewegende Jahre in der Außen- und Sicherheitspolitik liegen hinter uns. Die hier vorgelegte Bilanz gibt darüber Auskunft, was in den internationalen Politikbereichen, also der Außenpolitik, Sicherheitspolitik, Entwicklungszusammenarbeit und Menschenrechtspolitik, trotz zum Teil widriger Umstände erreicht wurde. Denn bei den zentralen Entscheidungen in Sachen Kosovo, Mazedonien und Afghanistan musste Rot-Grün mit sich selbst und vielen Kritikern in der Gesellschaft ringen, es mussten schwere und erklärungsbedürftige Entscheidungen getroffen werden. Aber Sozialdemokraten und Grüne haben zugleich versucht, ihre programmatischen Vorstellungen zur internationalen Politik - die sich natürlich im Diskurs über diese drei Hauptherausforderungen weiterentwickelten - Schritt für Schritt und richtungsgetreu umzusetzen. Und das nicht ohne Erfolg.

Ordnende Überschriften für die rot-grünen Anstrengungen im internationalen Bereich könnten so lauten:

- Mehr Prävention und bessere Fähigkeiten zur vorausschauenden Friedenspolitik als Alternative zu bewaffneten Interventionen.

- Handlungsfähigkeit der EU und eine umfassende Integrationsstrategie als der »europäische Weg« zum Frieden.

- Weiterentwicklung der Entwicklungs- und Menschenrechtspolitik zur globalen Friedenspolitik mit dem Ziel einer fairen und solidarischen Weltordnung als weltweite, strukturelle Präventionsstrategie gegen Hunger, Armut und Unterdrückung sowie daraus resultierendem Extremismus und Terrorismus.

Rot-Grün hat die Weichen für eine Außen- und Sicherheitspolitik gestellt, die sich eine nachhaltige Friedenssicherung zum Ziel setzt, die Konsequenzen aus den strukturellen Veränderungen der Globalisierung und ihren Folgen zu ziehen versucht und Prävention, auch im globalen Maßstab, zur Maxime erhebt. Natürlich wurden in diesen ersten vier Jahren einer rot-grünen Außen- und Sicherheitspolitik auch Fehler gemacht - vermeidbare und solche, die etwas mit den unerwartet dramatischen Prüfungen in Sachen Friedenspolitik zu tun hatten. Aber es ist gelungen, in unruhiger Zeit Richtung zu halten. Darauf kann man aufbauen.

\section{Friedenspolitik im Fegefeuer: Drei Zäsuren in vier Jahren}

\subsection{Kosovo - mit Krieg gegen die Gewalt}

Oktober 1998: Der rot-grüne Wahlsieg liegt erst wenige Tage zurück, da ereilt uns schon die bittere Realität. In Belgrad herrscht einer der letzten kaltblütigen Machthaber Europas, der die internationale Gemeinschaft immer wieder an der Nase herumführt und dabei buchstäblich über Leichen geht. Ungezählte Gespräche und Vermittlungsbemühungen sind wirkungslos verhallt. Milošević, besessen von seinen nationalistischen Machtphantasien, reagiert auf die Aktivitäten der separatistischen und ebenfalls vor Gewaltakten nicht zurückschreckenden UČK mit äußerster Brutalität und lässt die albanische Bevölkerung im Kosovo rücksichtslos demütigen, vertreiben und ermorden. Europa und die restliche Welt sind fassungslos über soviel Kaltblütigkeit und Zynismus, wie sie aus Belgrad zu vernehmen sind.

In dieser Situation entschließt sich die internationale Gemeinschaft zu einem dramatischen Schritt: Sie fordert Milošević ultimativ auf, diese Politik, die sich zu einer Krebsgefahr für ganz Südosteuropa entwickelt, zu unterlassen. Andernfalls, so droht sie, werde sie mit Hilfe der NATO militärisch intervenieren. Der Bundestag muss zu einer Sondersitzung zusammen kommen, um über eine eventuelle deutsche Beteiligung an militärischen Zwangsmaßnahmen zu entscheiden. Zwar geschieht dies in der alten Zusammensetzung, in der Union und FDP noch eine Mehrheit haben, aber die SPD-Bundestagsfraktion weiß, dass sie sich der Verantwortung nicht entziehen kann. Denn im Zweifel ist es die von ihr getragene Regierung, die wenige Tage später ihr Amt antreten wird, die diesen Schritt umsetzen und verantworten muss.

Der Druck auf Milošević zeigt Wirkung: Die Repressalien gegen die Kosovo-Albaner lassen nach. Erleichterung macht sich breit - zu früh, wie sich zeigt. Anfang 1999 dreht Milošević, provoziert durch neue UČK-Aktivitäten, die Daumenschrauben wieder an. Die Flüchtlingsströme wachsen wieder. Milošević kalkuliert, dass der Westen nur blufft. Noch einmal werden die roten Teppiche ausgerollt. Im französischen Rambouillet werden Serben und Albaner zu Verhandlungen gedrängt, eine politische Lösung zu finden. Am Ende vergeblich.

Alle diplomatischen Mittel scheinen ausgeschöpft. Was ist zu tun? Wenn der Westen jetzt nachgibt, hätte Milošević im Kosovo freie Hand. Das Leben hunderttausender KosovoAlbaner wäre akut gefährdet. Zudem hätte die NATO auf Jahre hinaus ihre Glaubwürdigkeit verspielt. Das im Oktober 1998 mit Gewaltanwendung verbundene Ultimatum reduziert die eigenen Optionen. Jetzt bleibt nur noch die Möglichkeit, den Beschluss vom Oktober 1998 umzusetzen und die 
Machthaber in Belgrad mit militärischen Mitteln zu zwingen, die grundlegenden Rechte der albanischen Bevölkerung im Kosovo zu respektieren. Um diese Ziele zu erreichen, sah die NATO mehrstufige und in der Intensität eskalierende Militärschläge vor, die je nach Widerstand Milošević durchaus von längerer Dauer sein konnten. In Europa war jedoch eine Hoffnung entstanden, dass wenige Tage ausreichen würden, Milošević in die Knie zu zwingen.

Leider erfüllt sich diese Hoffnung nicht. Milošević erweist sich als hartnäckig, die militärischen Erfolge gegen die serbische Armee bleiben gering, so dass der Krieg automatisch eskaliert. Bilder von Fehlschlägen, wie von der Zerstörung der chinesischen Botschaft in Belgrad oder von unschuldigen toten Zivilisten, bestimmen die Medienberichterstattung. Der Druck, zu einem Ende der Auseinandersetzungen zu kommen, nimmt von Tag zu Tag zu. In dieser Situation ist es die rot-grüne Bundesregierung, die initiativ wird und einen Weg aus der diplomatischen Sackgasse zu finden versucht.

Mit einem Fünf-Punkte-Plan wird Russland wieder ins Boot geholt und ein diplomatisches Ende des Kosovo-Krieges erreicht. Die Vereinten Nationen übernehmen jetzt wieder die Verantwortung. Die jugoslawische Armee muss sich vollständig aus dem Kosovo zurückziehen und die vertriebenen Kosovo-Albaner können zurück in ihre Heimat. Eine Fortsetzung des Krieges bis zur völligen Kapitulation Belgrads, wie von Teilen des Bündnisses beabsichtigt, wird nicht zuletzt durch die deutsche Initiative abgewendet. Die Entmachtung Milošević besorgt das serbische Volk später selbst, als es ihn im Herbst 2000 nach massiven Wahlfälschungen vom Sockel stürzt. Heute sitzt Milošević in Den Haag, wo er sich vor dem Internationalen Kriegsverbrechertribunal verantworten muss.

Nach Ende des Krieges wird unter Aufsicht der Vereinten Nationen der mühsame Versuch unternommen, die verfeindeten serbischen und albanischen Volksgruppen wieder miteinander zu versöhnen. Eine enorm große Herausforderung, die immer wieder von Rückschlägen begleitet ist, zu der es letztendlich aber keine Alternative gibt. Geleitet wird die UN-Mission mittlerweile von Michael Steiner, einem deutschen Diplomaten und ausgewiesenen Balkanexperten.

Ein weiterer wichtiger Baustein ist der ebenfalls auf deutsche Initiative zurückzuführende Stabilitätspakt für Südosteuropa. Mit ihm werden die Staaten der Balkanregion ermuntert, enger miteinander $\mathrm{zu}$ kooperieren, statt sich voneinander abzugrenzen. Der Stabilitätspakt bietet eine europäische Integrationsperspektive, die allen Staaten der Region eine Mitgliedschaft in der Europäischen Union in Aussicht stellt. Dieses Programm, zu dem Deutschland jährlich 150 Mio. Euro beisteuert und damit nach den USA zweitgrößter Einzelgeber unter den Einzelstaaten ist, hat maßgeblich zu einer Beruhigung der Situation auf dem Balkan beigetragen. Die SPD-Bundestagsfraktion begleitet diesen Prozess auf der parlamentarischen Ebene mit einer »Task Force Stabilitätspakt« und hat bereits mehrere Konferenzen mit Parlamentariern aus allen südosteuropäischen Staaten durchgeführt.

\subsection{Mazedonien - die waffengeschützte Friedensstrategie}

Wer geglaubt hatte, mit dem Ende des Kosovo-Krieges und der Etablierung des Stabilitätspaktes für Südosteuropa sei die Gewalt auf dem Balkan verschwunden, wird im Frühjahr 2001 eines Besseren belehrt. Der seit langem schwelende Konflikt zwischen Slavomazedoniern und Albanern beginnt langsam zu brennen. Angestachelt durch die Erfolge ihrer kosovo-albanischen Brüder radikalisieren sich nun auch albanische Extremisten in Mazedonien und werden zu gewalttätigen Freischärlern. Ihre Legitimation leiten sie aus der mangelhaften Repräsentanz der albanischen Bevölkerungsgruppe in staatlichen Institutionen ab sowie aus der Tatsache, dass versprochene Reformen seit Jahren verschleppt worden sind. Die sich daraus entwickelnde Unzufriedenheit fordert nun mit gewaltsamen Aktionen den mazedonischen Staat heraus.

Die Regierung Mazedoniens, das bis dahin als Insel politischer Stabilität galt, reagiert unverhältnismäßig hart. Es kommt zu zahlreichen Gefechten. Tote und Verletzte sind zu beklagen. Die Auseinandersetzung droht, auf die Gesamtheit der beiden Bevölkerungsgruppen, Slavomazedonier und Albaner, die bislang weitgehend friedlich zusammengelebt hatten, überzugreifen. In Europas Hauptstädten schrillen die Alarmglocken. Nach vier blutigen Balkankriegen, bei denen Europa nicht immer rechtzeitig und richtig reagiert hat, kann sich niemand vorstellen, diesmal tatenlos zuzuschauen, wie sich ein Staat selbst zerstört. Die europäische Krisendiplomatie funktioniert diesmal besser. Javier Solana, inzwischen Europas Mann für die Gemeinsame Außen- und Sicherheitspolitik, reist mehrfach nach Skopje, um mit den verfeindeten Konfliktparteien zu reden und sie zu einer politischen Lösung zu drängen.

Zusätzlich entsenden die EU und die USA jeweils einen Sondergesandten in die Region, um den diplomatischen Druck zu erhöhen. Im August 2001 ist es soweit: Die mazedonische Regierung hat sich nach wochenlangen Verhandlungen bereit erklärt, die längst überfälligen Staatsreformen einzuleiten und den Albanern mehr Rechte zuzubilligen. Im Gegenzug erklären sich die albanischen Kämpfer bereit, ihre Waffen abzugeben und sich in den politischen Prozess zu integrieren. Der gemeinsame und entschlossene Wille der Europäer und der USA, einen Bürgerkrieg unter gar keinen Umständen zuzulassen, hat Erfolg.

Voraussetzung für eine friedliche Lösung ist die von beiden Konfliktparteien gewünschte Kontrolle der NATO bei der Übergabe der albanischen Waffen. Die Vereinten Nationen begrüßen diesen Schritt und unterstützen die NATO in diesem Anliegen. Auch Deutschland soll sich an der sogenannten »Mission Essential Harvest« beteiligen. Dies setzt wieder einen Beschluss des Bundestages voraus.

Die Sommerpause 2001 ist geprägt von hitzigen Debatten über Sinn und Zweck eines solchen Einsatzes. Viele haben noch die Erfahrungen des Kosovo-Krieges vor Augen und fürchten eine ungewollte Eskalation. Alle Argumente und der Hinweis, dass sowohl beide Konfliktparteien als auch der UN-Sicherheitsrat geschlossen hinter dieser Maßnahme stehen, führen leider nicht dazu, dass die Koalition am Ende einheitlich abstimmt. Eine kleine Gruppe sozialdemokratischer und grüner Abgeordneter bleibt bei ihrem Nein. 
Dass schon wenige Zeit später kein Mensch mehr über Mazedonien und verfehlte eigene Mehrheiten sprechen wird, ahnt zu diesem Zeitpunkt niemand. Heute birgt das Thema Mazedonien keinen innenpolitischen Konfliktstoff mehr. Die Bundeswehr ist dort im Rahmen der NATO-Folgemission »Task Force Fox « stationiert und sorgt mit dafür, dass der eingeleitete Verständigungsprozess zwischen Mazedoniern und Albanern auf friedlichem Wege fortgesetzt wird.

\subsection{Der 11. September - das unfassbar Neue}

Bis zum frühen Nachmittag ist der 11. September in Berlin ein gewöhnlicher Sitzungstag in der Haushaltswoche des Bundestages. Das, was sich an diesem Tag in New York und Washington ereignet und live über die Fernsehbildschirme in alle Welt übertragen wird, übertrifft die bis dahin gängigen Vorstellungen von Terrorakten. Doch hier führt nicht Hollywood Regie, wie man im ersten Augenblick vielleicht noch annehmen konnte, sondern die anti-zivilisatorische Kraft eines islamistischen Fundamentalismus. Die Ziele dieses Angriffs sind nicht zufällig ausgewählt. Sie repräsentierten Amerikas Größe und Stolz. Eine Zerstörung dieser Insignien der Macht, so das Kalkül der Verbrecher, soll die »kulturelle Hegemonie « westlicher Werte zurückdrängen.

Doch diese Rechnung geht nicht auf. Der »Kampf der Kulturen«, auch im Westen von manch einem heraufbeschworen, findet nicht statt. Die Welt verharrt in den ersten Stunden und Tagen nach dem Anschlag in gespannter Erwartung, wie Amerika antworten werde. Doch das, was viele erwartet hatten, ein reflexartiger Gegenschlag ohne vorherige Konsultation der Vereinten Nationen und der Verbündeten, findet nicht statt. Statt dessen erleben wir eine amerikanische Administration, die strategisch klug und weitsichtig handelt Die Vereinten Nationen und die NATO werden eingeschaltet und um Unterstützung gebeten.

Bereits am 12. September werden in der Sicherheitsratsresolution 1368 die Anschläge als »Bedrohung des Weltfriedens und der internationalen Sicherheit « verurteilt und zugleich bekräftigt, »dass diejenigen, die den Tätern, Drahtziehern und Förderern helfen, sie unterstützen oder ihnen Zuflucht gewähren, zur Rechenschaft gezogen werden«. Damit setzt der Sicherheitsrat die Anschläge mit einem kriegerischen Angriff gleich und bestätigt dem angegriffenen Amerika das Recht auf Selbstverteidigung und legitimiert darüber hinaus auch ein Vorgehen gegen die Schützer der Attentäter, in diesem Fall das erratische Talibanregime in Afghanistan. In einer weiteren Resolution vom 28. September (1373) bestätigt der VN-Sicherheitsrat ohne jeden Zweifel das Selbstverteidigungsrecht der USA und die Zulässigkeit des Einsatzes »aller Mittel« gegen diese erfolgten Angriffe und die damit verbundene Bedrohung.

Es entspricht der Logik dieser Gleichsetzung von Terroranschlag und kriegerischem Angriff, auch die NATO um die Auslösung des Bündnisfalles zu ersuchen. Durch diplomatische Aktivitäten rund um den Globus gelingt es den Vereinigten Staaten, eine »Große Koalition gegen den Terrorismus « zusammenzubringen und dabei Staaten wie Russland und die Volksrepublik China, aber auch fast alle arabischen und islamisch geprägten Staaten miteinzubeziehen.
Erst nach diesen multilateral ausgerichteten Aktivitäten, nach wochenlangen Vorbereitungen und nach mehrfachen, allerdings erfolglosen Ultimaten an die Adresse der Taliban, beginnt am 7. Oktober 2001 die militärische »Operation Enduring Freedom «. Nach anfänglichen Schwierigkeiten gelingt es den Amerikanern, zusammen mit der »Nordallianz« die Herrschaft des Talibanregimes zu brechen und die Handlungsfähigkeit des Netzwerkes von Al Quaida deutlich einzuschränken.

Diese militärischen Erfolge bahnen den Weg für einen politischen Neuanfang in Afghanistan nach 22 Jahren Bürgerkrieg. Auf der Grundlage des »Bonn Agreement « vom 5. Dezember 2001 bildet sich eine multiethnische und politisch pluralistisch zusammengesetzte Übergangsregierung. Die wichtigsten politischen Kräfte des Landes stimmen der Übergangslösung und einem Stufenplan zur vollständigen Renormalisierung des politischen Lebens zu. Am 13. Juni 2002 wählt die »Loya Jirga « Interimspremier Hamid Karsai zum Präsidenten Afghanistans. In 18 Monaten sollen freie und demokratische Wahlen die Phase des Übergangs abschließen.

Die rot-grüne Bundesregierung setzt während der gesamten Zeit eigene politische Akzente im Kampf gegen den Terror: Mit der Autorität ihrer aktuellen Präsidentschaft in der »Afghanistan Support Group « ausgestattet geht die Bundesregierung mit einer Erhöhung ihrer Mittelzusagen zugunsten von Humanitärer Hilfe für die notleidende afghanische Zivilbevölkerung auf über 50 Mio. Euro voran und erreicht damit zahlreiche weitere Mittelaufstockungen, so zum Beispiel bei der EU auf über 250 Mio. Euro. Die Bundesregierung trägt dadurch dazu bei, dass die äußerst schwierigen Aufgaben der internationalen Hilfsorganisationen bei der Versorgung der afghanischen Bevölkerung und der anschwellenden Zahl von Flüchtlingen nicht an zu geringen Geldmitteln scheitern.

Am 16. November 2001 konkretisiert der Deutsche Bundestag nach langen und schwierigen Beratungen in den einzelnen Fraktionen und in Verbindung mit der vom Bundeskanzler gestellten Vertrauensfrage die bereits am 19. September angekündigte »Bereitstellung geeigneter militärischer Fähigkeiten zur Bekämpfung des internationalen Terrorismus«. Das Parlament stimmt der Entsendung von bis zu 3.900 Soldaten im Rahmen der »Operation Enduring Freedom« zu. Es sind vorwiegend defensive Fähigkeiten, die Deutschland bereitstellt: Sanitätskräfte, Lufttransportkräfte, ABC-Abwehrkräfte, Seestreitkräfte und sogenannte Spezialkräfte, die vor Ort vor allem zur Ergreifung von Tätern aus dem Al Quaida-Netzwerk unter schwierigen Umständen oder für Geiselbefreiungen vorgesehen sind. Was die Beteiligung an »Enduring Freedom« angeht, legen die Fraktionen von SPD und Grünen größten Wert auf eine geographische Einschränkung. In dem Antragstext heißt es: »Deutsche Kräfte werden sich an etwaigen Einsätzen gegen den internationalen Terrorismus in anderen Staaten als Afghanistan nur mit Zustimmung der jeweiligen Regierung beteiligen.«

Dahinter steckt das Bestehen auf dem »Täterbezug« bei der deutschen Beteiligung am Antiterrorkampf. Das wird noch einmal in der zum Beschluss gehörenden Protokollerklärung der Bundesregierung verdeutlicht, die zum Auftrag der Soldaten erläutert, dass die »genannten Operationsziele sich allein gegen das terroristische Netzwerk Bin Ladens, Al Quaida, und diejenigen, die es beherbergen und unterstützen, 
richten«. Damit ist ein Riegel vor eine Ausweitung der Antiterroroperationen ohne eine neue Beschlussfassung des Deutschen Bundestages geschoben.

Die beiden Koalitionsfraktionen machen in einem parallel verabschiedeten Entschließungsantrag deutlich, dass der Kampf gegen den Terrorismus allein mit militärischen Mitteln nicht gewonnen werden kann. Er zeichnet die Umrisse einer globalen Strategie zur Eindämmung der verhängnisvollen Kettenreaktion von Armut und Demütigung über Hass und Gewaltbereitschaft bis zu Gotteskriegertum und Terrorismus. SPD und Grüne bestehen darauf, dass die westlichen humanitären Grundsätze auch im Kampf gegen den Terror gelten und dass im militärischen Einsatz vor Ort »das Prinzip der Verhältnismäßigkeit und der größtmöglichen Vermeidung ziviler Opfer« Beachtung findet.

Die Entschließung des Bundestages weist auf die Notwendigkeit hin, die gefährlichen regionalen Konflikte vom Nahen Osten bis Kaschmir so rasch wie möglich zu entschärfen, die Anstrengungen für eine Verbesserung ziviler Konfliktbearbeitung und Krisenprävention zu erweitern, sich neuen politischen Strategien zur Humanisierung der Globalisierung und zur Korrektur einer ungerechten und unfairen Weltordnung zu widmen, den Dialog der Kulturen zu intensivieren und die Vereinten Nationen als wichtigste Instanz für die Verrechtlichung der internationalen Beziehungen zu stärken.

Die deutsche Politik schaltet sich besonders intensiv in die internationalen Bemühungen um die politische Zukunft Afghanistans ein. Seine Krönung findet dieses Engagement in der Konferenz der Vereinten Nationen zur Zukunft Afghanistans, die am 26. November 2001 auf dem Bonner Petersberg beginnt und am 5 . Dezember mit der international »Bonn Agreement « genannten Friedens- und Übergangsvereinbarung erfolgreich abschließt. Die Beendigung des 22 Jahre anhaltenden Bürgerkrieges, das Öffnen eines Weges in eine stabile Zukunft Afghanistans, die Bildung der Übergangsregierung Karsai und der Plan für eine schrittweise Renormalisierung des politischen Lebens in einer zweijährigen Übergangsphase - all das ist ein großer Erfolg der Vereinten Nationen, es verbindet sich aber auch mit den besonderen deutschen Vermittlungsbemühungen.

Am 22. Dezember 2001 beschließt der Bundestag, diesmal ohne Kontroverse, einen deutschen Beitrag zu der bereits auf dem Petersberg beschlossenen Internationalen Sicherheitsunterstützungstruppe für die Übergangsregierung in Afghanistan (International Security Assistance Force/ISAF).

Im Januar 2002 gibt die Bundesregierung auf der internationalen Geberkonferenz in Tokio die Zusage, Afghanistan in den kommenden vier Jahren mit 320 Mio. Euro zu unterstützen. Andere Nationen erklären sich ebenfalls zu einer umfassenden Aufbauhilfe bereit. Damit sind noch lange nicht alle Probleme in Afghanistan gelöst. Die ungeklärte Sicherheitsfrage behindert die Normalisierung des Lebens im Lande und bedroht auch den vereinbarten Übergang in eine demokratische $\mathrm{Zu}$ kunft. Dennoch gibt es zu dem eingeschlagenen Weg keine Alternative. Afghanistan wird noch auf lange Sicht unserer Unterstützung bedürfen.

\section{Grundlagen deutscher Außen- und Sicherheitspolitik: Multilateralismus aus Prinzip}

»Die Bundesregierung wird sich mit aller Kraft um die Entwicklung und Anwendung von wirksamen Strategien und Instrumenten der Krisenprävention und der friedlichen Konfliktregelung bemühen «, so steht es in der Koalitionsvereinbarung zwischen SPD und Bündnis 90/Die Grünen vom 20. Oktober 1998. Im April 2000 hat die Bundesregierung ihr entsprechendes Gesamtkonzept vorgelegt. Darin bekennt sie sich zu den im Koalitionsvertrag benannten Zielen. Ausgangspunkt für Maßnahmen der Krisenprävention und der Konfliktbeilegung ist ein erweiterter Sicherheitsbegriff, der politische, ökonomische, ökologische und soziale Stabilität umfasst.

Eine Reihe konkreter Maßnahmen wurden eingeleitet: So bietet das Auswärtige Amt seit 1999 spezielle Kurse zur Vorbereitung auf internationale Missionen an. Ein Personalpool von über 500 zivilen Experten wurde geschaffen und für Friedensmissionen der VN sowie für Missionen und Krisenreaktionskräfte der OSZE genutzt. Um den angestrebten hohen Standard zu halten, hat ein eigens zu diesem Zweck gegründetes Zentrum für Internationale Friedensmissionen (ZIF) im Juli 2002 seine Arbeit aufgenommen.

Die Zusammenarbeit mit Nichtregierungsorganisationen wurde ausgebaut und gezielt zivilgesellschaftliche Projekte und Maßnahmen der Gewaltprävention, Konfliktbearbeitung und Konflikttransformation, die auf eine konstruktive Veränderung der Gesamtbedingungen eines Konfliktes zielen, gefördert.

Die Europäische Sicherheits- und Verteidigungspolitik hat sich seit dem Amtsantritt von Rot-Grün spürbar weiter entwickelt. Seit Oktober 1999 hat die europäische Außenpolitik mit Javier Solana ein eigenes Gesicht. Es waren nicht zuletzt die Erfahrungen des Kosovo-Krieges, die den Ausbau der ESVP vorantrieben. Die Präsenz und die Handlungsfähigkeit der europäischen Außenpolitik hat sich in vielen Bereichen, insbesondere auch in Krisensituationen, verstärkt. Namentlich auf dem Balkan, u. a. während der Mazedonienkrise, aber auch im Nahen Osten tritt die EU zunehmend als eigenständiger Akteur auf, der mit einer Stimme spricht.

Ab dem Jahr 2003 wird die EU in der Lage sein, begrenzte militärische Operationen zur Krisenbewältigung durchzuführen. Um bestehende Defizite im militärischen Bereich abzubauen und durch Synergieeffekte eine höhere Effizienz zu erreichen, müssen die Verteidigungsanstrengungen und die Rüstungskooperation auf europäischer Ebene zukünftig noch stärker koordiniert werden.

Auf der zivilen Seite wird die EU im Bedarfsfall 5.000 Polizisten zur Verfügung stellen können. Deutschland stellt mit über 900 Polizisten den zweitgrößten Anteil. Anfang 2003 wird die EU die Nachfolge der bisher von den Vereinten Nationen geführten Polizeimission in Bosnien und Herzegowina übernehmen. Dies wird dann die erste zivile ESVP-Operation sein.

Besonderen Stellenwert legen Sozialdemokraten auf die parlamentarische Begleitung dieses Prozesses. Aus diesem Grund hat die SPD in der zurückliegenden Legislaturperiode eine umfassende Konferenzserie mit den sozialdemokratischen 
Schwesterparteien in den EU-Mitgliedsstaaten zu diesem Themenbereich durchgeführt.

Mit Polen, Tschechien und Ungarn wurden 1999 drei neue Mitglieder in die NATO aufgenommen. Auf dem NATOGipfel in Prag im November 2002 steht die Aufnahme weiterer Mitglieder an. Es ist gut möglich, dass aus den heute 19 Mitgliedsstaaten in absehbarer Zeit 26 Mitglieder werden, die der NATO zwangsläufig ein neues Gesicht geben werden.

Auch der 11. September hat das atlantische Bündnis verändert. Die NATO hat mit der Ausrufung des Bündnisfalles entschlossen und konsequent reagiert. Die Bedeutung des Bündnisses in der Zukunft wird nicht zuletzt davon abhängen, ob es ihm gelingt, die enormen Herausforderungen in eine neue umfassende Strategie einfließen zu lassen. Die herkömmliche Rolle als ein Verteidigungsbündnis im euro-atlantischen Raum scheint angesichts neuer, asymmetrischer Bedrohungen abzunehmen.

Die Bedrohung mit Massenvernichtungswaffen durch unberechenbare Staaten oder terroristische Organisationen ist leider nicht von der Hand zu weisen. Das Phänomen der sogenannten privatisierten Gewalt, d.h. die zunehmende Bedrohung durch nicht-staatliche Akteure, dringt mehr und mehr in unser Bewusstsein. Darüber zu entscheiden, wie man diesen Bedrohungen am wirksamsten begegnet, ist Aufgabe der Politik.

Eine wirksame Abschreckungs- und Verteidigungsfähigkeit ist auf jeden Fall erforderlich. Für Sozialdemokraten war jedoch immer klar, dass sich die Antworten nicht in militärischen Optionen erschöpfen dürfen. Soziale Ungerechtigkeit und Perspektivlosigkeit insbesondere in den Ländern der Dritten Welt bilden immer auch den Resonanzboden für Gewaltbereitschaft. Daher ist es unser primäres Anliegen, die strukturellen Ursachen von Gewalt zu beseitigen.

Für die SPD ist die Stärkung multilateraler Organisationen wie $V N$ und $O S Z E$ oberstes Prinzip. Institutionen wie die OSZE erfüllen durch ihre präventive, übergreifend kooperative, auf Krisenfrüherkennung und rechtzeitigen zivilen Interessenausgleich ausgerichtete Arbeit eine unverzichtbare Aufgabe.

Die OSZE spielt eine bedeutende Rolle in den Konfliktregionen im Kaukasus und in Zentralasien. Insbesondere in den Staaten der letztgenannten Region ist eine dauerhafte Arbeit für Frieden, Sicherheit und Menschenrechte ohne die Mitwirkung der OSZE nicht denkbar.

Die zivilgesellschaftsfördernde Rolle der OSZE-Institutionen ist unbestritten. Mit ihren Langzeitmissionen, den Konsultations- und Dialogmöglichkeiten im Rahmen des Ständigen Rates, den verschiedenen Frühwarnmechanismen verfügt die OSZE inzwischen über gute Instrumente auf dem Gebiet der Konfliktverhütung. Einen Schwerpunkt ihrer Arbeit leistet sie mit ihren Feldaktivitäten und dem Aufbau einer funktionierenden Zivilgesellschaft. Diese Rolle bedarf erheblicher zusätzlicher Anstrengungen, die insbesondere von den Mitgliedstaaten getragen werden müssen.

Eine von Rot-Grün getragene Bundesregierung wird die politische Rolle der OSZE für die friedliche Konfliktprävention und für die demokratische Stabilität weiterhin deutlich herausstellen und ihre Aufgaben und Ziele unverändert wirkungsvoll unterstützen.

Die SPD ist davon überzeugt, dass die Vereinten Nationen als globale Organisation zur Herstellung und Wahrung des Friedens sowie zur Bewältigung globaler Herausforderungen stärker genutzt werden müssen. Dazu müssen sowohl die finanziellen Grundlagen als auch die administrativen und politischen Strukturen gestärkt werden. Deutschland hat in den zurückliegenden Jahren eine aktive Rolle in zahlreichen Bereichen der UN-Politik gespielt; dazu gehören z. B. die finanziellen Leistungen als drittgrößter Beitragszahler, die Unterstützung des Anti-Minen Abkommens von Ottawa, der Kampf gegen Proliferation von Kleinwaffen, die Beteiligung am Stand-by-System für friedenserhaltende Maßnahmen, die Ausbildung von Fachkräften für zivile Friedensmissionen und bei der Einrichtung des Internationalen Strafgerichtshofes.

Die Hauptaufgabe der Vereinten Nationen besteht unverändert darin, eine zentrale Rolle bei der Herbeiführung und Wahrung des Weltfriedens und der internationalen Sicherheit zu spielen. Dies erfordert eine größere Bereitschaft der Mitgliedstaaten, Abrüstungsinitiativen der Vereinten Nationen, krisenpräventive, friedensschaffende und friedenserhaltende Maßnahmen zu unterstützen. Abrüstung, Rüstungskontrolle und die Stärkung der Nichtverbreitungsregime sind unverzichtbare Elemente der Konfliktprävention, Konfliktbeilegung und der Friedenskonsolidierung und Bestandteil kooperativer Sicherheitspolitik.

Die SPD hält die vertragliche Abrüstungs- und Rüstungskontroll- und Nichtverbreitungspolitik weiterhin für einen Grundpfeiler internationaler Sicherheit. Sie hat dies in einem gemeinsamen Koalitionsantrag Anfang Juni 2002 noch einmal zum Ausdruck gebracht.

Pläne, wie sie in den USA diskutiert werden, Nuklearwaffen als operative einsetzbare Instrumente in die Militärstrategie zu integrieren, würden die Schwelle zur potenziellen Anwendung nuklearer Waffen deutlich senken und eventuell Staaten, die bislang keine eigene nukleare Bewaffnung besitzen, ermuntern, von diesem Weg abzuweichen. Bereits heute sind Entwicklungen zu beobachten, die Anlass zur Sorge geben. Indien und Pakistan setzen ihre Pläne zur nuklearen Bewaffnung weiter um. Die immer wieder aufflammenden Spannungen zwischen den beiden Ländern zeigen die Dringlichkeit, das weltweite nukleare Waffenkontrollregime zu stärken.

Auch vor dem Hintergrund des Kampfes gegen den internationalen Terrorismus müssen die Anstrengungen zur weiteren Abrüstung und Rüstungskontrolle sowie zur Verhinderung der Weiterverbreitung von Massenvernichtungswaffen und entsprechender Trägermittel intensiviert werden. Multilaterale Instrumente, Rüstungsexportkontrolle, internationale Kooperation und politischer Dialog sollten dabei im Vordergrund stehen.

Frieden, Sicherheit und Stabilität können dauerhaft nicht durch einseitige Schutzsysteme oder durch den Aufbau und Einsatz von militärischen Offensivpotenzialen erreicht werden, sondern letztlich nur durch Kooperation und Partnerschaft, Vertrauensbildung und multilaterale, verifizierbare Abkommen zur Abrüstung, durch Rüstungskontrolle und Nichtverbreitung. 


\section{Rot-grüne Entwicklungspolitik - Umsteuern in Zeiten der Haushaltskonsolidierung}

Der Kassensturz der rot-grünen Bundesregierung nach dem Wahlsieg 1998 übertraf die schlimmsten Befürchtungen. Statt aus dem Vollen schöpfen zu können, musste überall der Rotstift angesetzt werden, um den drohenden finanziellen Kollaps Deutschlands abzuwenden. Alle Ressorts müssen ihren Beitrag dazu leisten. Dies ist insbesondere dann schmerzhaft, wenn es - wie im Falle des BMZ - ein Ministerium trifft, das die ärmsten Länder dieser Welt im Kampf gegen Hunger und Unterentwicklung unterstützen soll.

Trotz der schwierigen Rahmenbedingungen gelang es, auch in diesem Bereich sichtbare Akzente zu setzen. Heidemarie Wieczorek-Zeul hat der Entwicklungspolitik wieder ein politisch erkennbares Gesicht gegeben. Rot-Grün hat erstmals die Entwicklungspolitik als gesamtpolitische Querschnittsaufgabe etabliert und durch zahlreiche Maßnahmen die Voraussetzungen für Kohärenz verbessert. Das BMZ erhielt wichtige neue Zuständigkeiten, die es ermöglichen, die Entwicklungspolitik konsistenter als bislang zu gestalten.

Einer der Schwerpunkte ist die Halbierung der weltweiten Armut bis zum Jahr 2015. Deutschland hat als eines der ersten Länder im April 2001 ein Aktionsprogramm zur Erreichung dieses Ziels beschlossen und damit internationale Beachtung gefunden. Bereits 1999 wurde auf dem Kölner G8-Gipfel mit deutscher Unterstützung eine Entschuldungsinitiative für die ärmsten und am stärksten verschuldeten Länder vereinbart.

Die Entschuldungsinitiative ist ein gutes Beispiel für das neue entwicklungspolitische Verständnis, das nationale und internationale Reformen miteinander verbindet. Gute Regierungsführung und Armutsbekämpfung in den betroffenen Ländern sind unabdingbare Voraussetzung für eine Entschuldung auf internationaler Ebene. Damit ist die Entschuldungsinitiative als Hebel genutzt worden, um Armutsbekämpfung in den Entwicklungsländern, aber auch bei IWF und Weltbank politische Priorität zu geben. Die Bundesregierung hat sich gemeinsam mit anderen für eine Reform von IWF und Weltbank eingesetzt, weg von wirtschaftszentrierten Strukturanpassungsprogrammen hin zu einer Politik, die die Bekämpfung der Armut und die Lebensbedingungen der Menschen in den Entwicklungsländern in den Mittelpunkt stellt.

Mit dem Zivilen Friedensdienst hat Rot-Grün ein innovatives Instrument geschaffen, das den gewaltfreien Umgang mit Konflikten und Konfliktpotentialen fördern soll. Allein in den ersten zwei Jahren wurden über 120 Friedensfachkräfte entsandt, die vor Ort Beiträge zur Mediation in schwelenden Konflikten, zur Versöhnung und zur Verarbeitung von Traumata leisten.

Seit dem Regierungswechsel setzt sich die Bundesregierung verstärkt für die Berücksichtigung der Belange der Entwicklungsländer in Handelsvereinbarungen ein. Das Abkommen von Cotonou auf europäischer Ebene im Jahr 2000 zeigt, dass ein Ausgleich unterschiedlicher Interessen von Entwicklungsund Industrieländern auch im Handelsbereich unter Stärkung gemeinsamer Werte wie Menschenrechte, Demokratie und Good Governance möglich ist.

Im März 2002 fand in Monterrey die internationale Konferenz Financing for Development der Vereinten Nationen statt. Zur Vorbereitung dieser Konferenz hat das BMZ eine Studie zur Durchführbarkeit einer Devisentransaktionssteuer (»Tobinsteuer«) in Auftrag gegeben, so wie dies auch in einem gemeinsamen Antrag von SPD und Grünen gefordert wird. Die Ergebnisse liegen bereits vor und sind durchaus ermutigend.

Einen neuen »Global Deal« zwischen Industrie- und Entwicklungsländern durchzusetzen, lautet das Ziel. Frieden und Wohlstand sind nicht teilbar. Fragen nach Demokratie, offenem interkulturellem Dialog und sozialer Gerechtigkeit werden lauter denn je gestellt. Hier liegen staatliche Gestaltungsaufgaben, die mit Hilfe der Entwicklungspolitik angegangen werden müssen. Die rot-grüne Bundesregierung hat mit ihrer Politik in den vergangenen vier Jahren ihren Anteil dazu beigetragen.

Trotz des Haushaltskonsolidierungsprogramms halten wir an dem Ziel fest, bis zum Jahr 2006 0,33 Prozent des Bruttosozialprodukts für die Entwicklungshilfe zur Verfügung zu stellen. Mit dem vorgelegten Haushaltsentwurf für das Jahr 2003, der eine Steigerung des BMZ-Etats um 2,7 Prozent vorsieht, wird dieser Weg konsequent fortgesetzt. Die Zusammenarbeit mit den Nichtregierungsorganisationen im Bereich der Entwicklungshilfe ist für uns von großer Bedeutung. Dies zeigt sich auch im Regierungsentwurf für den Bundeshaushalt 2003. Das BMZ wird seine Finanzmittel zur Förderung der Zivilgesellschaft um 53,7 Mio. Euro, von 381 Mio. auf 434 Mio. Euro, erhöhen - das entspricht einer Steigerung von mehr als 14 Prozent.

Im Januar 2000 verabschiedete das Kabinett die neuen »Politischen Grundsätze für den Export von Kriegswaffen und sonstigen Rüstungsgütern « und löste damit die alte, aus dem Jahr 1982 stammende Regelung ab. Die Fraktionen von SPD und Grünen waren an der Neuformulierung der Grundsätze unmittelbar beteiligt und konnten auf diese Weise ihre Vorstellungen einbringen. Bereits unmittelbar nach der Regierungsübernahme wurde das Ministerium für wirtschaftliche Zusammenarbeit und Entwicklung (BMZ) neu in den Bundessicherheitsrat aufgenommen, in dem über Anträge zum Rüstungsexport entschieden wird.

Die neuen Grundsätze verlangen bei Exportentscheidungen ein »besonderes Gewicht « für die Beachtung der Menschenrechtssituation im Empfängerland und schließen Waffenexporte in Länder aus, in denen »fortdauernde und systematische Menschenrechtsverletzungen « stattfinden. Waffenlieferungen in Länder, die nicht Mitglied der NATO (bzw. ihr gleichgestellte Länder wie Schweiz, Japan, Australien, Neuseeland) sind, werden grundsätzlich restriktiv gehandhabt, wobei erstmalig auch ernsthafte Beeinträchtigungen nachhaltiger Entwicklung als Kriterium genannt werden. Besonderen Wert legt die Neufassung auf eine strikte Sicherung des Endverbleibs gelieferter Waffen.

Neu ist auch das Instrument eines jährlichen Rüstungsexportberichts der Bundesregierung, der im Parlament debattiert wird und damit die Kontrollmöglichkeiten des Bundestages über die Rüstungsexportpolitik erweitert. Im zweiten von der rot-grünen Bundesregierung vorgelegten Rüstungsexportbericht für das Jahr 2000 ist im Bereich der Kriegswaffenlieferungen ein Rückgang von 53 Prozent gegenüber dem Vorjahr 
zu verzeichnen. Sie machten nur 0,11 Prozent aller deutschen Exporte aus.

Der Wert der Rüstungsgüter, für die Genehmigungen zum Export in Drittländer (d. h. Länder, außerhalb von NATO/EU und der gleichgestellten Länder) erteilt wurden, gingen im Vergleich zum Vorjahr um 24 Prozent zurück. Seit 1998 haben sich diese Zahlen sogar nahezu halbiert (1998: 2,02 Mrd. Mark, 2000: 1,17 Mrd. Mark).

\section{Menschenrechte - Schritte nach vorn statt Sonntags- reden}

»Achtung und Verwirklichung der in der Allgemeinen Erklärung der Menschenrechte proklamierten und in den Menschenrechtsverträgen festgeschriebenen Menschenrechte sind Leitlinien für die gesamte internationale Politik der Bundesregierung « - so steht es in der Koalitionsvereinbarung vom Herbst 1998.

Mit der Stelle eines Menschenrechtsbeauftragten im Auswärtigen Amt sowie mit dem Haushaltstitel »Unterstützung von Maßnahmen zur Förderung der Menschenrechte « hat RotGrün die operativen Möglichkeiten für eine aktive Menschenrechtspolitik ausgebaut. Zum ersten Mal in seiner Geschichte hat der Deutsche Bundestag auch einen eigenständigen Ausschuss für Menschenrechte gebildet. Damit unterstreicht er auch institutionell die wachsende Bedeutung der Menschenrechte in der politischen Praxis. Eines der weiteren wichtigsten Projekte rot-grüner Menschenrechtspolitik war die Gründung eines Deutschen Instituts für Menschenrechte. Das Institut wird die Menschenrechtsarbeit im In- und Ausland wirksamer machen. Die Stärkung des internationalen Menschenrechtsschutzsystems ist ein Grundpfeiler der Menschenrechtsarbeit der Koalition. Wichtigstes Ereignis ist hier zweifellos, dass das Römische Statut in Kraft getreten ist und der Internationale Strafgerichtshof in Den Haag demnächst seine Arbeit aufnehmen wird. Die Bundesrepublik Deutschland hat das Statut für den Strafgerichtshof von Anfang an unterstützt und die gesetzlich notwendigen Voraussetzungen dafür geschaffen. Um so bedauerlicher ist es, dass sich die Vereinigten Staaten diesem wichtigen Schritt bislang verweigern und die Einrichtung des Strafgerichtshofs boykottieren.

Die beste Menschenrechtspolitik ist jene, die gewaltsame Konflikte vermeiden hilft. Wir fördern diesen Politikansatz nach Kräften. Deshalb haben wir den Zivilen Friedensdienst ins Leben gerufen und beteiligen uns an zahlreichen friedenserhaltenden und friedensstiftenden Maßnahmen. Schwerpunkte unseres Friedens-Engagements befinden sich in Zentralamerika, im östlichen Afrika, in Afghanistan oder auf dem Balkan. Auch unsere neuen Richtlinien für eine restriktive Rüstungsexportpolitik wirken in diesem präventiven Sinne.

Durch die Globalisierung sind insbesondere die wirtschaftlichen, sozialen und kulturellen Rechte stärker ins Blickfeld gerückt. Lange führten sie neben den politischen und bürgerlichen Rechten eher ein Schattendasein. Ihre Gleichrangigkeit ist jedoch unbestritten. Die Verantwortung der Wirtschaft für die Menschenrechte spiegelt sich in der vor kurzem unterzeichneten gemeinsamen Erklärung von Regierung, Wirtschaft, Gewerkschaften und Nichtregierungsorganisationen über den Schutz der Menschenrechte wider.

Die Bundesrepublik Deutschland engagiert sich in zahlreichen internationalen und europäischen Gremien für die Menschenrechte. Im Verbund mit den anderen EU-Staaten nutzt sie ihr politisches Gewicht regelmäßig bei der jährlichen Tagung der Menschenrechtskommission der Vereinten Nationen in Genf. Auf deutsche Initiative hin wurde das Budget des Europäischen Gerichtshofs für Menschenrechte massiv erhöht, damit dieser den mit der Zahl der Mitglieder im Europarat enorm gewachsenen Aufgaben besser gerecht werden kann.

Die Bundesregierung hat wesentlich die Inhalte der Grundrechtecharta der Europäischen Union mit geprägt, die die Grund- und Menschenrechte in der EU stärkt. Der nächste Schritt, die Erarbeitung einer europäischen Verfassung, ist bereits eingeleitet. Die genannten Beispiele zeigen: Menschenrechtspolitik hat in dieser Legislaturperiode einen qualitativen Sprung getan. Diesen Weg wollen wir konsequent fortsetzen.

\section{Die Bundeswehr zukunftsfähig machen}

Als Rudolf Scharping vor vier Jahren das Amt des Verteidigungsministers antrat, blickte die Bundeswehr zurück auf ein Jahrzehnt der verpassten Gelegenheiten. Die Kohl-Regierung hatte es versäumt, die Bundeswehr konzeptionell und strukturell auf die neuen Herausforderungen des 21. Jahrhunderts einzustellen. Auf die rot-grüne Koalition kam die überfällige Aufgabe $\mathrm{zu}$, die Bundeswehr zu reformieren und sie damit zukunftsfähig zu machen. Zusätzlich wurde sie im Laufe der letzten vier Jahre mit neuen Aufgaben konfrontiert, die zu Beginn der Legislaturperiode nicht einmal in Ansätzen erkennbar waren.

Nur zum Vergleich: Im Oktober 1998 befanden sich weniger als 3.000 Soldaten der Bundeswehr im Auslandseinsatz die meisten von ihnen in Bosnien-Herzegowina. Heute ist die Zahl 10.000 überschritten. Viele von ihnen auf dem Balkan (Bosnien, Kosovo und Mazedonien), ein kleiner Teil in Georgien und - wie bereits ausführlich behandelt - im Rahmen von ISAF und OEF in Afghanistan, im östlichen Mittelmeer, auf der arabischen Halbinsel und am Horn von Afrika. Rotation, Vorausbildung und Nachbereitung binden fast 50.000 weitere Soldaten in Deutschland.

Moderne und zeitgemäß ausgerüstete Streitkräfte sind unverzichtbarer Bestandteil eines leistungsfähigen Staates. Mit der eingeleiteten »Erneuerung von Grund auf « - der umfassendsten Reform in der Geschichte der Bundeswehr wird ein entscheidender Beitrag geleistet, um die außen- und sicherheitspolitische Leistungsfähigkeit Deutschlands in den kommenden Jahren sicher zu stellen.

Die Reform der Personalstruktur ist ein entscheidender Eckpfeiler der Bundeswehrreform. Künftig werden der Bundeswehr ca. 285.000 Soldaten angehören. Mit der personellen Neuausrichtung wird die Bundeswehr zukünftig ihre Aufgaben weit besser als heute wahrnehmen können. 
Die Maßnahmen zur Verbesserung der Ausrüstung der Bundeswehr sind nicht kurzfristiger Natur. Um langfristige Planungssicherheit zu gewährleisten, wurde der Etat in der mittelfristigen Finanzplanung für die kommenden vier Jahre auf 23,62 Mrd. Euro verstetigt und vor dem Hintergrund der Ereignisse des 11. September um 767 Mio. Euro dauerhaft aufgestockt. Schließlich ist es gelungen, die Investitionsquote der Bundeswehr, die unter der Kohlregierung zuletzt auf 21,6 Prozent gesunken war, wieder auf 24,5 Prozent anzuheben. Dieser Weg soll auch in den kommenden Jahren kontinuierlich fortgesetzt werden.

Das Thema Wehrpflicht ist ein viel diskutiertes Thema in Deutschland. Der Versuch von Gegnern der allgemeinen Wehrpflicht, diese auf juristischem Wege auszuhebeln, ist im April 2002 gescheitert. Das Bundesverfassungsgericht hat mit seinem einstimmigen Urteil deutlich gemacht, dass die Wahl zwischen Wehrpflicht oder Berufsarmee Sache des Gesetzgebers ist. Es kommt daher darauf an, die Debatte wieder $\mathrm{zu}$ versachlichen.

Der Behauptung, die heutige sicherheitspolitische Lage Deutschlands erübrige eine Wehrpflicht, erteilten die Karlsruher Richter eine Absage. Neben verteidigungspolitischen Gesichtspunkten müssen auch allgemeinpolitische, wirtschaftsund gesellschaftspolitische Gründe bewertet und gegeneinander abgewogen werden, so das BVerfG. Auch bestehende Bündnisverpflichtungen spielen in diesem Zusammenhang eine wesentliche Rolle.

Die SPD-Bundestagsfraktion hat die Diskussion über die Wehrpflicht nie zu einem Tabu erhoben. Die Wehrpflicht muss sich immer wieder neu legitimieren. Die Kritiker müssen jedoch anerkennen, dass sich die Wehrpflicht seit Bestehen der Bundeswehr bewährt hat. Die Bundeswehr ist heute fest in unserer Gesellschaft verankert. Sie genießt hohes Ansehen und leistet hervorragende Arbeit. Diese Errungenschaften leichtfertig aufs Spiel zu setzen, wäre unverantwortlich. Die Dauer des Grundwehrdienstes ist heute so niedrig wie nie zuvor. Sie beträgt seit Januar 2002 nur noch neun Monate.

Erfahrungen anderer Länder zeigen, dass sich Hoffnungen, durch die Abschaffung der Wehrpflicht Kosten einzusparen, nicht erfüllt haben. Eine Armee, die ausschließlich auf Freiwillige angewiesen ist, verfügt zwangsläufig über ein sehr viel eingeschränkteres Bewerberreservoir. De facto gewinnt die Wehrpflichtarmee mit geringerem Aufwand besser qualifiziertes Personal, da über die allgemeine Wehrpflicht die gesamte Bandbreite schulischer und beruflicher Qualifikationen unserer Gesellschaft in die Streitkräfte hineinkommen.

\section{Zukunftsoption »Nachhaltiger Frieden«}

Am 28. Februar 2002 trat der Generalsekretär der Vereinten Nationen erstmals mit einer Rede im Deutschen Bundestag auf. Nicht zufällig sprach er fast ausschließlich über Afghanistan. Der Friedensnobelpreisträger warb eindringlich dafür, sich langfristig vor Ort zu engagieren, nicht zu früh rauszugehen und Afghanistan zu einem Beispiel für einen neuen Ansatz von »sustainable peace « zu machen. Die Strategie einer auf Dauerhaftigkeit angelegten Friedenspolitik stellte Kofi Annan gleichberechtigt neben das Postulat der »Nachhaltigen Entwicklung«, das sich weltweit immer stärker durchsetzt.
Und er warnte vor den Fehlern der Vergangenheit, als es zu früh zur »Vernachlässigung « von Konflikten kam, statt zur Beherzigung der Regel »no exit without strategy«, also kein Abzug, ohne über ein dauerhaftes Stabilisierungskonzept zu verfügen. Für den UN-Generalsekretär entscheidet sich am Beispiel Afghanistan die Frage der Glaubwürdigkeit der westlichen Antworten auf die Herausforderung des Terrorismus.

Mit Recht hat Kofi Annan darauf gezählt, dass seine Botschaft in Berlin auf offene Ohren stieß. Diese Bundesregierung zeigt die Bereitschaft, daran mitzuwirken, jene globalen Verwerfungen zu beseitigen, die den Nährboden für Extremismus und Terrorismus bilden. Deswegen ist das politische Deutschland auch offen für jeden vernünftigen Vorschlag in dieser Richtung.

Wir stehen am Anfang eines neuen Kapitels der Weltpolitik: Auf dem Balkan mussten die Europäer lernen, dass ihre Fähigkeiten zur regionalen Krisenprävention nicht ausreichten und sie dafür bitteres Lehrgeld zu zahlen hatten. Am Beispiel der Mazedonienkrise wird deutlich, dass sich diese Fähigkeiten inzwischen verbessert haben. Aber schon stehen wir einer völlig neuen Herausforderung gegenüber: Man könnte sie als Aufgabe zur Herausbildung von Instrumenten und Fähigkeiten zur strukturellen oder globalen Prävention bezeichnen.

In Europa zweifelt niemand daran, dass der Kampf gegen die Netzwerke und Verstecke des Terrorismus im Innern wie im Äußern fortgesetzt werden muss. Aber wir haben die Pflicht, jeden einzelnen Schritt an seiner Wirkung im Gesamtkontext der Terrorismusbekämpfung zu messen und abzuwägen.

Niemand hat heute fertige Konzepte. Es gibt jedoch eine Reihe viel versprechender Ansätze bei der Behandlung von regionalen Konflikten, bei der Antwort auf gefährliche Entstaatlichungstendenzen, bei der besseren Verteilung von globalen Entwicklungschancen, bei der humaneren Gestaltung der Globalisierung und bei der notwendigen Intensivierung des Dialogs der Kulturen. Diese Ansätze müssen am Ende in eine schwerpunktmäßig zivile Strategie der strukturellen und globalen Prävention gegen die terroristischen Aktivitäten einfließen.

Es könnte einem schwindelig werden, wenn man die letzten zwei Jahre Revue passieren lässt und sich einmal die Geschwindigkeit anschaut, mit der wir vor immer neue Herausforderungen gestoßen werden, $\mathrm{zu}$ denen die alten Antworten nicht mehr passen wollen. Der 11. September stellt dabei einen Kulminationspunkt dar, bleibt aber Glied einer Ereigniskette, die hier auch nicht endet. Bei solchen Erlebnissen tauchen bei den Menschen, den betroffenen wie den beobachtenden, ungezählte Fragen auf. Und damit wächst der Erwartungsdruck an die Politik, Orientierung zu geben.

Wie schon beschrieben, hat die amerikanische Politik auf die ungeheuerliche Demütigung und Verwundung zunächst in besonnener und entschlossener Weise reagiert. Gemeint ist das multilaterale Vorgehen, das Anrufen von UN und NATO, die Bildung der weltweiten politischen Koalition gegen den Terror, die sorgfältige Vorbereitung der Militäraktionen gegen Al Quaida und Taliban, die schon wegen des Schutzbedürfnisses der Gesellschaft gegen Wiederholungstaten aus der Sicht des angegriffenen Landes alternativlos waren. 
Doch viele Fragen, deren Beantwortung für die Zukunft entscheidend sein wird, konnten noch nicht beantwortet werden: Aus welchen Strukturen heraus konnten die Attentäter überhaupt ausgebildet und die Terroranschläge vorbereitet werden? Mit welchen zivilen und mit welchen militärischen Maßnahmen lassen sich ähnliche Attacken, wie die vom 11. September, verhindern? Über welche Optionen verfügen wir? Wie können die ungeheuren Spannungen im Nahen Osten und den angrenzenden Regionen, die auch Ausdruck einer sozialen Modernisierungskrise der islamischen Welt sind, in einen friedlichen Evolutionsprozess umgewandelt werden, der den Menschen eine konkrete Perspektive in der Welt des 21. Jahrhunderts eröffnet?

Wir müssen versuchen, Antworten auf diese Fragen zu entwickeln. Die Menschen erwarten dies von der Politik. Dabei landen wir ganz schnell bei der Weltordnung, die wir selber auf diesem Planeten zulassen. Das Nachfragen führt uns zu den globalen Tendenzen der Entstaatlichung, Privatisierung und Kommerzialisierung der Gewalt (Erhard Eppler), die uns längst in zahlreichen Weltregionen die größten Probleme bereiten. Dann müssen wir klären, ob es sich hierbei um die Auswirkungen einer Globalisierung handelt, die staatliche Gewalt schwächt, den Abstieg von Regionen und ganzen Subkontinenten (etwa des südlichen Afrika) schulterzuckend zur Kenntnis nimmt und bei der Reparaturfrage auf die Selbstheilungskräfte des Marktes setzt.

Die Zerbröselung des staatlichen Gewaltmonopols kennzeichnet heute keineswegs nur vergessene Drittweltregionen, dieses Geschwür wuchert längst in die fortgeschrittensten Industriestaaten hinein. Wo der Neoliberalismus solange gegen den Staat agiert hat, bis diesem die nötigen Mittel zur Aufrechterhaltung seines Gewaltmonopols verweigert werden, da entsteht eine neue Klassengesellschaft: Wer sich Sicherheit nicht kaufen kann, bleibt ohne Schutz und es entsteht jene »Zitadellengesellschaft«, die alle Konflikte und Probleme der gespaltenen Weltgesellschaft im überschaubaren Raum von Stadt und Land getreulich abbildet. Die alten Rezepte des Strukturkonservatismus, des Neoliberalismus oder gar des Populismus bleiben hier stumm oder führen geradewegs in die Sackgasse.

In der Welt von heute greifen Außen- und Innenpolitik genauso wie Sicherheits- und Entwicklungspolitik, Handels-, Finanzpolitik immer mehr ineinander. Und es ist noch nicht ausgemacht, wer sich die Definitionsmacht für diese Neue Zeit erobern wird. Dies allein ist Grund genug, für eine Fortsetzung von Rot-Grün über den 22. September hinaus zu kämpfen.

\section{Überlegungen zur Zukunft der Transatlantischen Beziehungen ${ }^{1}$}

Dr. Karl Lamers, MdB, außenpolitischer Sprecher der CDU/ CSU-Bundestagsfraktion, Berlin

1) Der Angriff des transnationalen Terrorismus vom 11. September 2001 auf die Symbole der militärischen und wirtschaftlichen Macht Amerikas - das Pentagon und das WTC - ist der radikalste Ausdruck der Auflehnung gegen die von den USA als dem Protagonisten des Westens geprägte Weltordnung. Er ist Zeichen ihrer tiefen Krise. Der Beifall für dieses monströse Ereignis bei den Massen in der islamischen Welt, aber auch Reaktionen in anderen ihrer Teile sind das eigentlich politisch Beunruhigende. Der Westen - nicht nur seine Vormacht Amerika - ist herausgefordert. Er muss eine grundlegende Neubewertung des Zustandes, in dem sich die Welt befindet, und eine Neubestimmung seiner Verantwortung für sie vornehmen, um die unerlässlichen militärischen und anderen operativen Maßnahmen in einen ebenso unerlässlichen, aber noch nicht vorhandenen, (definierten) politischen Rahmen stellen zu können. Der 11. September 2001 demonstriert die Verletzlichkeit der heutigen Zivilisation, und dass auch Amerika verwundbar

1 Rede am 27. Juni 2002. ist. Der Westen muss sich neu formieren und die tiefen Differenzen überwinden, die mit dem 11. September offenkundig wurden.

Der 11. September 2001 hat in brutaler Deutlichkeit die Notwendigkeit, ja die Unausweichlichkeit eines noch engeren europäisch-amerikanischen Zusammenwirkens gezeigt. $\mathrm{Zu}$ diesem Zweck müssen Europa und Amerika vorab und vor allem das ganze Ausmaß des Aufruhrs gegen die heutige Weltordnung und dessen Ursachen wahrnehmen, es sich bewusst machen, die Herausforderung annehmen, ihre Politik davon bestimmen lassen, alle Politik - die innere wie die äußere - und vor allem die Prioritäten anders setzen: Außenpolitik hat Vorrang, weil unser Wohl und Wehe, das der Völker des Westens, immer mehr vom Wohl und Wehe aller unserer Nachbarn abhängt und Nachbar sind heute alle.

Der konkrete Ausdruck der Prioritäten seiner Interessen ist der Haushalt eines Landes. In Deutschland sind die Ausgaben für auswärtige Zwecke, soweit sie in den Haushalten für das Auswärtige Amt, die Entwicklungshilfe und die Verteidigung zum Ausdruck kommen, seit 1990 bis 2002 von mehr als ca. 21 Prozent auf weniger als ca. 12 Prozent am Gesamthaushalt zurückgegangen. 